\title{
Construcción de un esquema silvícola para Nothofagus dombeyi, usando área potencial aprovechable, índice de máxima densidad y variación del crecimiento diametral según densidad
}

\author{
Construction of a silvicultural scheme for Nothofagus dombeyi using potential available area, \\ maximum density index and diameter growth variation according to density
}

\author{
Pablo Cruz a*, Alejandro Bascuñán ${ }^{\text {a }}$ \\ *Autor de correspondencia: ${ }^{a}$ Universidad Mayor, Escuela de Ingeniería Forestal, Oterra, Camino la Pirámide 5750, \\ Huechuraba, Santiago, Chile, tel: 02 23281367, tacora1@gmail.com
}

\begin{abstract}
SUMMARY
This study proposes a silvicultural management scheme for Nothofagus dombeyi considering a combination of Reineke density index, the punctual density estimation with the method available potential area (APA) and a new function created to calculate the diametric growth when this is depending on density. The Index of maximum density allowed defining the minimum density before losing a land area and onset density for tree crown competence. Considering this information, it proposes a silvicultural scheme, applying tree cut for density reduction and keeping the forest in a rank established to obtain a better diameter of growing with minimum interventions. The Scheme included cleaning (2) and thinning (4), from 23 to 81 years old, with a residual density of 340 tree hectare $^{-1}$, and a diameter at breast height $(\mathrm{DBH})$ near $44 \mathrm{~cm}$. The application of this scheme would offer a wood volume of $757 \mathrm{~m}^{3}$ hectare-1 total by tree cut added to residual volume at the moment of reaching $50 \mathrm{~cm}$ of DBH. This would mean $1.217 \mathrm{~m}^{3}$ hectare ${ }^{-1}$ in 86 years, and would generate a middle diametric growth of $14.1 \mathrm{~m}^{3}$ hectare $^{-1}$ year $^{-1}$.
\end{abstract}

Key words: Nothofagus dombeyi, Reineke’s density index, available potential area, density guide.

\section{RESUMEN}

En este estudio se propone un esquema de manejo silvícola para Nothofagus dombeyi, a partir de la combinación del índice de densidad de Reineke, la estimación de densidad puntual con el método del área potencial aprovechable (APA) y la creación de una función de incremento diametral dependiente de la densidad. El índice de máxima densidad permitió definir las densidades mínimas antes de subutilización del sitio, y de la densidad de inicio de la competencia de copa. Con estas estimaciones se propuso un esquema silvícola, aplicando cortas de reducción de densidad de manera que el bosque se mantuviera en los rangos descritos, para obtener el mejor crecimiento diametral con el mínimo número de intervenciones. El esquema incluyó dos clareos y cuatro raleos, desde los 23 años de edad y hasta los 81 años en que la densidad residual debiera ser de 340 árboles ha ${ }^{-1}$ y un DAP cercano a 44 cm. La aplicación del esquema ofrecería un volumen total de $757 \mathrm{~m}^{3} \mathrm{ha}^{-1}$ durante el período, el que sumado al volumen residual al momento de alcanzar el DAP $50 \mathrm{~cm}$, significarían un volumen de $1.217 \mathrm{~m}^{3} \mathrm{ha}^{-1}$ en todo el período de 86 años, y generarían un crecimiento medio de $14,1 \mathrm{~m}^{3}$ ha $^{-1}$ año $^{-1}$.

Palabras clave: Nothofagus dombeyi, índice de densidad de Reineke, área potencial aprovechable, guía de densidad.

\section{INTRODUCCIÓN}

Una de las herramientas silvícolas más frecuentes y sencillas de usar es la regulación de la densidad. En contraste a ello, existen fuertes vacíos de conocimientos respecto de cuáles son los valores óptimos de densidad en que se debería mantener cada tipo de bosque para lograr maximizar los efectos de la silvicultura. En bosques de especies intolerantes, que tienden a ser coetáneos y puros o compuestos de pocas especies (Vita 1996), se facilita la interpretación de la competencia, dado que la mayor parte de los individuos están en similares estratos verticales del bosque y usando similares recursos del sitio. Posiblemente sea esta la razón de la profusa publicación de trabajos que utilizan la relación diámetro/densidad para bosques coetáneos a escala mundial (Reineke 1933, Cottam y Curtis 1956, Brown 1965, Gingrich 1967), de la que han surgido herramientas de sencilla aplicación como son los índices de densidad y guías de densidad.

Una guía de densidad es una herramienta que permite conocer para un diámetro cuadrático medio, los límites máximos de densidad, los de inicio de la competencia de copa y los de inicios de pérdida de producción potencial por baja densidad del bosque (Prodan et al. 1997), que operativamente puede representarse en gráficas cuyos ejes corresponden al área basal y a la densidad, o en algoritmos matemáticos. 
Diversos autores han construido guías de densidad o diagramas de manejo, combinando dos trabajos: el índice de máxima densidad de Reineke (1933) y los niveles de máxima competencia de Long (1985). Reineke (1933) postuló que la relación diámetro/densidad en bosques puros coetáneos, puede expresarse en una función lineal de doble logaritmo con un exponente universal de -1,605.

En el caso que el bosque evaluado tuviera un diámetro medio de $25 \mathrm{~cm}$, el índice de Reineke equivaldría a la densidad, ahora bien, si el bosque en cuestión fuera aquel que ha logrado la máxima densidad, se habría obtenido el valor máximo del índice de Reineke (IDR ${ }_{\max }$ ). Así, una manera de utilizar este concepto es encontrar empíricamente el valor de $\mathrm{IDR}_{\text {máx }}$, a partir de una serie de inventarios y que idealmente se ejecutarán en bosques con alta densidad. Obtenido dicho valor, es posible fijar el IDR como constante en la función y así reconstruir una serie completa de pares diámetro/densidad en que dicha densidad sería la máxima. Si bien el algoritmo ha sido usado profusamente en su fórmula original para diversas especies (Reque 2002, Woodall et al. 2005, Vacchiano et al. 2008), algunos investigadores han propuesto una especificidad del exponente con la especie, y, en consecuencia, recomiendan que se precise el exponente en cada caso (Adame et al. 2008, Navarro et al. 2011). Gezan et al. (2007) en particular propusieron el exponente -1,4112 para un grupo de especies del género Nothofagus, y Chauchard et al. (2001) proponen el exponente -1,622 para Nothofagus dombeyi (Mirb) Oerst. (coihue).

En años posteriores, Long (1985) estableció dos principios relacionados con el crecimiento basado en las máximas densidades de Reineke. El primero fue que el inicio de la competencia de copas por exceso de densidad ocurre en torno al $60 \%$ de la densidad máxima, y el segundo que el inicio de la pérdida de productividad potencial por baja densidad ocurre al 30 \% de la densidad máxima. Así, la combinación del $\mathrm{IDR}_{\max } \mathrm{y}$ los hitos de Long, permiten proponer una banda en la densidad dentro la que sería ideal mantener el bosque si se desea no perder potencial de crecimiento diametral. En Chile, Gezan et al. (2007) propusieron un cuarto nivel de densidad para el caso de Nothofagus spp., fijando un nivel mínimo de densidad no deseado como igual o menor a $20 \%$.

Un componente metodológico esencial de la construcción de índices de densidad es la manera de obtener la densidad para cada muestra de diámetro normal. Para estos estudios, es fundamental la localización de bosques con valores de densidad lo más alto posibles. Los inventarios tradicionales o llamados inventarios de masa, incorporan ruido a la construcción de índices diámetro/densidad, debido, por una parte, a que la parcela puede no ser pura, y a que, por otra, al analizar el atributo de un árbol, como el crecimiento diametral, por ejemplo, se le asocie la densidad de la parcela, y que no necesariamente se corresponda con la condición del árbol elegido. Un tercer componente de ruido es el usar inventarios realizados para otros fines y que no necesariamente se establecieron en zonas de alta densidad. Chauchard et al. (2001) y Gezan et al. (2007), por ejemplo, indican que al usar inventarios tradicionales para la elaboración de sus guías, tuvieron que aceptar parcelas que no eran puras, o que probablemente estaban lejos de las máximas densidades. Estos inconvenientes pueden superarse usando mediciones de densidad puntual, que significa determinar la densidad a la que está creciendo el árbol del que provienen las mediciones específicas. Para determinar una densidad puntual, Brown (1965) propuso la construcción de un polígono en torno al árbol muestra que se construye al cortar las distancias entre el sujeto muestra y cada vecino en porciones equidistantes, de manera que en el punto de corte se traza un rayo perpendicular al trazo entre los dos árboles. La intersección de todos los trazos permite crear un polígono que contiene el área potencial aprovechable (APA) del árbol muestra. Prodan et al. (1997) modificaron el método para ajustar la posición de los lados del polígono en función de la talla de cada árbol vecino. Usando la metodología de APA es posible obtener una relación diámetro/densidad específica para la condición de cada árbol muestra.

El objetivo del presente estudio fue proponer un esquema silvícola para Nothofagus dombeyi, a partir de la utilización combinada de la determinación de áreas potenciales de aprovechamiento (APA), el índice de densidad de Reineke, y funciones de incremento diametral. La hipótesis de trabajo, es que la combinación del uso de Reineke en su versión original combinada con la determinación de densidad usando APA, permiten construir esquemas de manejo para bosques coetáneos y puros como el trabajado en este estudio.

\section{MÉTODO}

Área de estudio. La zona se encuentra a $290 \mathrm{~km}$ al norte de la ciudad de Coyhaique (coordenadas UTM 5.135.200 m de latitud Sur y 717.725 m de longitud Este). El clima se caracteriza por ser de tipo oceánico, con bajas temperaturas, con abundantes precipitaciones, fuertes vientos y mucha humedad. La precipitación promedio anual fluctúa entre los 3.000 y $4.000 \mathrm{~mm}$. Los suelos son de tipo trumao, jóvenes, fuertemente modificados por la última glaciación (Cruces et al. 1999). Los bosques de la zona pueden clasificarse dentro del tipo forestal siempreverde ya que están dominados principalmente por Nothofagus dombeyi, Saxegothea conspicua (Lindl.) (mañío hembra) y Laurelia philliappiana (Looser) (tepa).

Colecta de información. El estudio se hizo en bosques puros de Nothofagus dombeyi, en los que se identificaron 45 áreas de bosque con la mayor densidad observada y de estructura coetánea, cuidando que el conjunto contuviera la mayor representación de diámetros hasta un máximo de $50 \mathrm{~cm}$. Dentro del bosque fue seleccionado un individuo objetivo, al que le fue medido su diámetro a la atura del pecho (DAP), su altura total y el crecimiento periódico de 
los últimos 5 años. Además se midió la distancia, Azimut y el DAP de cada uno de los "competidores cercanos", considerándose cercano aquellos dentro del área de influencia de una distancia horizontal equivalente a una altura del árbol objetivo.

Determinación de la densidad puntual, índice de densidad y construcción de la guía de densidad. Los datos de posición del árbol objeto y sus vecinos fue transformada a coordenadas cartesianas con el siguiente algoritmo (Cruz 2011):

$\mathrm{Xi}=\mathrm{Lij}^{*} \operatorname{sen} \alpha ; \mathrm{Yi}=\mathrm{Lij}^{*} \cos \alpha$

Donde:

$\mathrm{X}_{\mathrm{i}}$ : coordenada $\mathrm{x}$ para el árbol vecino $\mathrm{j}$.

$\mathrm{Y}_{\mathrm{i}}$ : coordenada y para el árbol objetivo i.

Lij: distancia entre el árbol objetivo y el árbol vecino i (metros).

$\alpha$ : azimut transformado a radianes.

Esta transformación permitió elaborar cada uno de los polígonos APA en el software ArcInfo, de manera sencilla $\mathrm{y}$, una vez construidos los polígonos, determinar la densidad puntual (árboles ha ${ }^{-1}$ ) de cada árbol muestra, según la expresión (figura 1):

$l_{i j}=\left(\frac{d_{i}}{d_{i}+d_{j}}\right) * L i j$

Donde:

lij = distancia entre árbol sujeto i y la perpendicular con el árbol $\mathrm{j}$.

$\mathrm{Lij}=$ distancia entre árbol sujeto $\mathrm{i}$ y el competidor $\mathrm{j}$.

di = diámetro del árbol sujeto i.

dj = diámetro del árbol competidor $\mathrm{j}$.
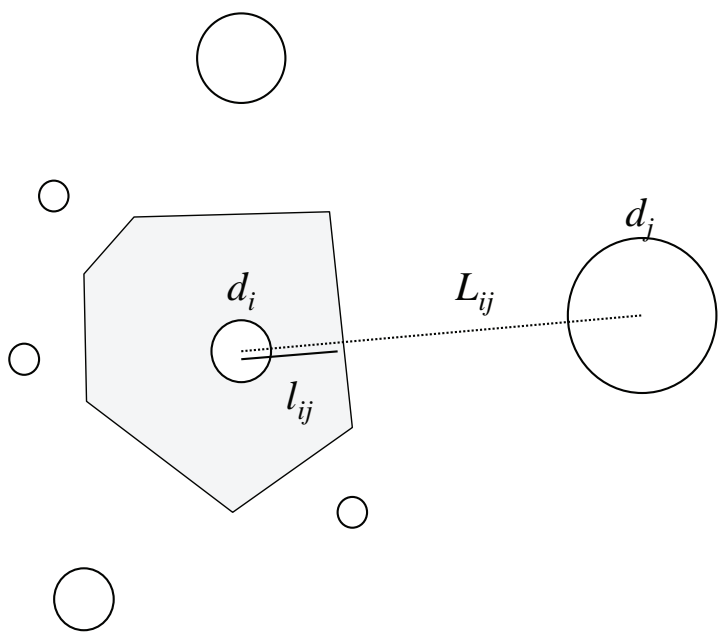

Figura 1. Representación del área potencial aprovechable (APA) y la creación de los polígonos de influencia.

Representation of available potential area and creation of polygons of influence.
A partir del APA, se determinó la densidad puntual de cada individuo, permitiendo la estimación del IDR para los árboles muestras.

Para definir el IDR máximo de coihue (IDR ${ }_{\max }$ ), fueron promediados los diez máximos. Es importante señalar que no existen estudios que recomienden un procedimiento para establecer el IDR $\max _{\text {x }}$ y para este estudio se propone un promedio de los máximos para amortiguar posibles errores en el muestreo o situaciones anómalas en el bosque.

La función de Reineke fue modificada, incorporando el $\mathrm{IDR}_{\max }$ como constante y dejando densidad como variable dependiente del diámetro medio, según la expresión:

$\mathrm{N}=\mathrm{IDR}_{\max } *\left(\frac{\mathrm{Dm}}{\mathrm{Dg}}\right)^{-1,605}$

Donde:

N: Densidad del rodal (árboles ha-1).

$\mathrm{IDR}_{\max }$ : Índice de densidad del rodal máximo.

Dm: Diámetro medio fijado (normalmente $25 \mathrm{~cm}$ ).

Dg: Diámetro medio del rodal (cm).

A partir de esta nueva función se obtuvo una serie con las densidades máximas para cada diámetro desde $5 \mathrm{~cm}$ y hasta $50 \mathrm{~cm}$. Aplicando la norma de Long (1985), se agregaron las densidades a $60 \%$ y a $35 \%$ de las máximas densidades determinadas ( $\mathrm{DN}_{60 \%} \mathrm{y} \mathrm{DN}_{35 \%}$, respectivamente), aumentando en $5 \%$ la mínima de Long para proponer una densidad mínima conservadora respecto de la original. Estas tres series de densidades constituyeron las curvas que permitieron confeccionar la guía de densidad.

Determinación del incremento periódico anual del diámetro. La determinación del incremento periódico anual (IPA $\left.{ }_{\text {dap }}\right)$ se hizo mediante la extracción de un tarugo con taladro de incremento a cada árbol objetivo seleccionado y su posterior evaluación de los últimos cinco anillos de crecimiento. Al ser un terreno sin pendiente, la extracción del tarugo se realizó tomando como referencia el Norte magnético y expresando el crecimiento en $(\mathrm{cm}$ año-1 $)$. Así, sobre la base de los 45 registros, se probaron los modelos potencial, logarítmico, exponencial y polinómico, usando como variable independiente la densidad y como variable dependiente el incremento, para seleccionar aquel con mejor desempeño estadístico.

Esquema de manejo silvícola. Obtenida la guía de densidad y la relación entre el IPA dap $y$ la densidad, según clase diamétrica, se propuso un esquema de manejo basado en dichas relaciones, y cuyo objetivo era maximizar los crecimientos individuales del diámetro. Así, fue analizada la evolución del crecimiento y la densidad de un bosque teórico con las herramientas descritas, donde se fueron asignando cortas para bajar la densidad, bajo los siguientes criterios:

1. Se propuso la aplicación de una intervención cuando la condición densidad-DAP del bosque generara una relación equivalente a un $\mathrm{DN}_{60 \%}$ como máximo. 
2. Todas las intervenciones dejarán el bosque con una densidad equivalente de un $\mathrm{DN}_{35 \%}$. Para confeccionar una propuesta práctica, las densidades residuales propuestas no serán exactas, sino que se redondearán a unidades de centenas, en vez de los valores exactos que arrojan las ecuaciones usadas.

3. Definida la nueva densidad según el paso anterior, se asume que el bosque aumenta en diámetro, según el modelo de incremento diametral creado. Para los años siguientes a la intervención se recalcula cada parámetro con las nuevas condiciones.

4. Las cortas teóricas afectan todo el rango de diámetros que el bosque tiene en ese momento, simulando un raleo con "criterio selectivo", de manera que el DAP del bosque residual no varía.

5. Se asumió una mortalidad natural del bosque de un 1 $\%$ anual.

Para establecer las condiciones iníciales del bosque teórico se propuso lo siguiente:

- La primera densidad usada se estimó promediando las densidades a $\mathrm{DN}_{100 \%}, \mathrm{DN}_{60 \%}$ y $\mathrm{DN}_{35 \%}$ para la clase diamétrica $5 \mathrm{~cm}$.

- La edad inicial se estableció estimando el tiempo para alcanzar la condición DAP $=5 \mathrm{~cm}$ con la función de incremento diametral, con la densidad establecida en el criterio anterior.

Para obtener los volúmenes totales y por consiguiente poder estimar los incrementos periódicos anuales del volumen $\left(\right.$ IPA $_{\text {vol }}$ ) se utilizó la función [4] para la especie Nothofagus dombeyi, obtenida del compendio de funciones dendrométricas del bosque nativo (Drake et al. 2003). La función de altura [5] usada fue extraída del trabajo de Donoso et al. (1999).

$V=E X P\left(-10,312929+0,991795 * L N *\left(D A P^{2} * H\right)\right)[4]$

$H=1,3+4,34559 * L N(D A P)$

Donde:

$\mathrm{V}$ : volumen total del fuste $\left(\mathrm{m}^{3}\right)$.

DAP: diámetro a la altura del pecho $(\mathrm{cm})$.

H: altura total (m).

Las variables incluidas en el esquema de manejo fueron: año de intervención (años), edad del bosque (años), clase de DAP $(\mathrm{cm})$, densidad inicial y densidad final (árboles ha-1), IDR (\%), crecimiento diametral ( $\mathrm{cm}$ año-1), volumen acumulado $\left(\mathrm{m}^{3} \mathrm{ha}^{-1}\right)$, actividad silvícola y extracción $\left(\mathrm{m}^{3} \mathrm{ha}^{-1}\right)$.

\section{RESULTADOS}

Determinación de la densidad puntual, índice de densidad y construcción de la guía de densidad. El promedio de los diez máximos valores de IDR encontrados a partir del cálculo de densidad con APA, fue de 2.387 (cuadro 1), el que a su vez permitió establecer las densidades máxima, inicio de autorraleo e inicio de competencia de copas según los criterios de Long (cuadro 2) para una serie de DAP comprendida entre los $5 \mathrm{~cm}$ y $50 \mathrm{~cm}$ (figura 2).

En este rango de valores fue posible estimar que la máxima densidad para bosques de coihue en la zona alcanzaría los 31.600 árboles por hectárea a los $5 \mathrm{~cm}$ de DAP, y que a diámetros de $50 \mathrm{~cm}$ podrían tener una densidad máxima de 785 árboles por hectárea.

Cuadro 1. Valores máximos de IDR según las densidades puntuales estimadas por el área potencial aprovechable para Nothofagus dombeyi.

IDR Maximum values, according to the specific densities estimated by the potential for available area for Nothofagus dombeyi.

\begin{tabular}{|c|c|c|c|}
\hline $\begin{array}{l}\text { DAP Árbol } \\
\text { muestra (cm) }\end{array}$ & $\mathrm{APA}\left(\mathrm{m}^{2}\right)$ & $\begin{array}{c}\text { Densidad } \\
\text { (árboles ha-1) }^{-1}\end{array}$ & $\begin{array}{c}\text { IDR } \\
\text { máximos }\end{array}$ \\
\hline 30 & 3,49 & 2.865 & 3.839 \\
\hline 31 & 3,94 & 2.538 & 3.585 \\
\hline 32 & 6,33 & 1.580 & 2.348 \\
\hline 41 & 9,65 & 1.036 & 2.292 \\
\hline 28 & 5,25 & 1.905 & 2.285 \\
\hline 41 & 9,69 & 1.032 & 2.283 \\
\hline 42 & 10,61 & 943 & 2.167 \\
\hline 23 & 4,99 & 2.004 & 1.753 \\
\hline 49 & 16,98 & 589 & 1.734 \\
\hline 35 & 10,83 & 923 & 1.585 \\
\hline
\end{tabular}

Cuadro 2. Densidades estimadas para los diferentes niveles propuestos por Long (1988), para clases diametrales de $5 \mathrm{~cm}$.

Densities estimated for different levels proposed by Long (1988), for $5 \mathrm{~cm}$ diametric classes.

\begin{tabular}{crrr}
\hline \multicolumn{4}{c}{ Densidad (Reineke) } \\
\hline Clase $(\mathrm{cm})$ & $\mathrm{DN}_{100 \%}$ & $\mathrm{DN}_{60 \%}$ & $\mathrm{DN}_{35 \%}$ \\
\hline 5 & 31.602 & 18.961 & 11.061 \\
10 & 10.389 & 6.233 & 3.636 \\
15 & 5.419 & 3.252 & 1.897 \\
20 & 3.415 & 2.049 & 1.195 \\
25 & 2.387 & 1.432 & 835 \\
30 & 1.782 & 1.069 & 624 \\
35 & 1.391 & 835 & 487 \\
40 & 1.123 & 674 & 393 \\
45 & 929 & 558 & 325 \\
50 & 785 & 471 & 275 \\
\hline
\end{tabular}


Guía de Densidad

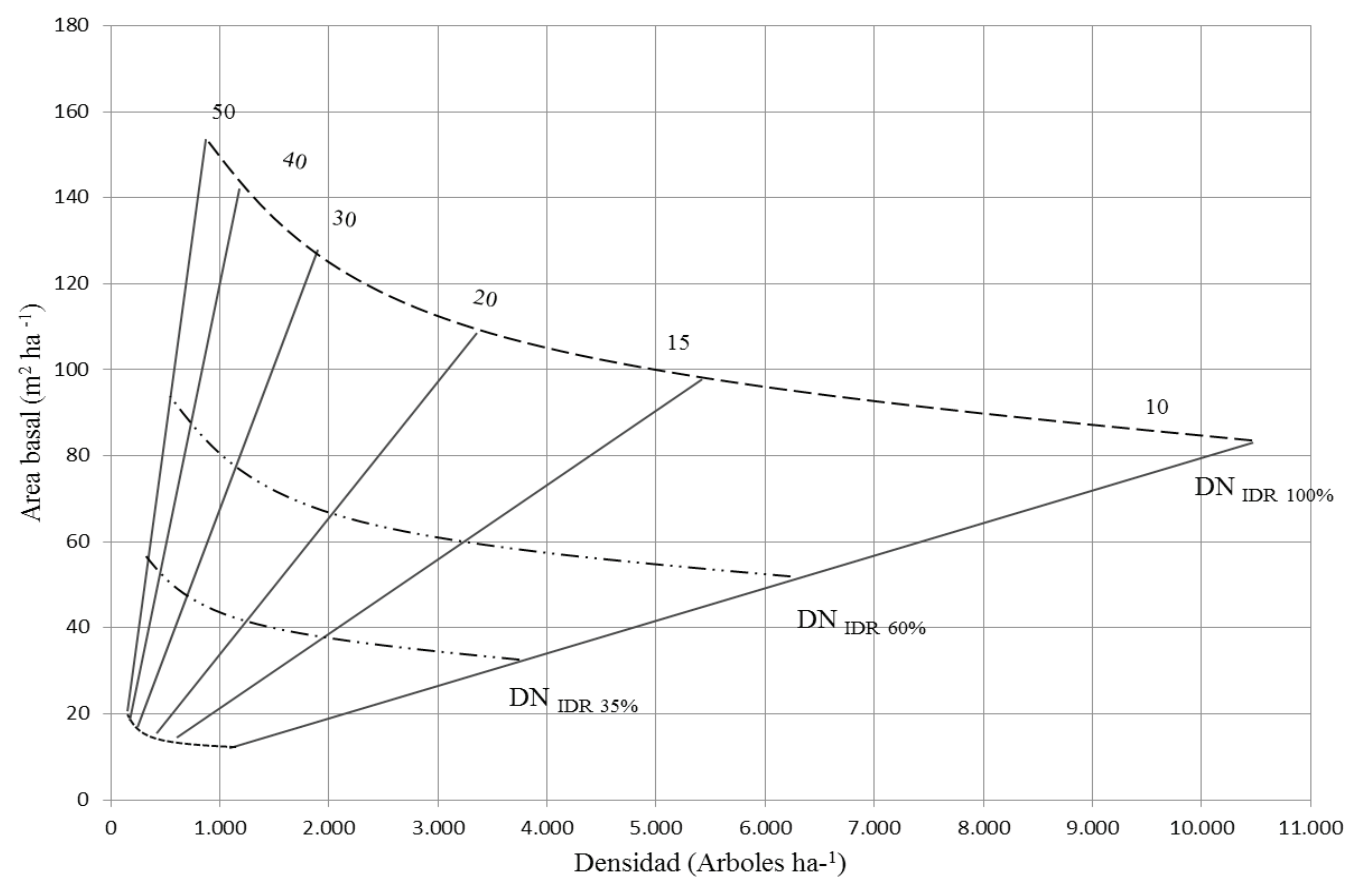

Figura 2. Guía de densidad para Nothofagus dombeyi expresando los niveles de densidad usados.

Density guide of Nothofagus dombeyi expressing the three density levels used.

Incremento periódico anual del diámetro. El modelo potencial representó la mejor función para estimar el IPA dap $_{\text {al }}$ obtenerse el mayor coeficiente de determinación $\left(\mathrm{R}^{2}=0,76\right.$; $P<0,001$ ) (figura 3), representado por la ecuación [6].

$\mathrm{IPA}=16,0407 * d^{-0,4271}$

Donde:

IPA: incremento periódico del DAP $(\mathrm{cm})$. d: densidad (árboles ha-1).

Con este modelo fue posible definir los incrementos diametrales según clase diamétrica y la densidad (cuadro 3, figura 4), que en general significa un aumento en torno al $55 \%$ en la densidad $\mathrm{DN}_{35 \%}$ respecto de la máxima densidad, para todos los casos.

Esquema de manejo silvícola. Dada las condiciones iniciales propuestas para comenzar el esquema, el manejo se comenzaría en un bosque de 20.500 árboles ha-1 ${ }^{-1}$ con un volumen acumulado de $135 \mathrm{~m}^{3}$ ha $^{-1}$ y 20 años de edad aproximada. Los criterios definidos para determinar el momento y la intensidad de cada actividad silvícola para la propuesta de manejo (cuadro 4) permitieron establecer un esquema silvícola de dos clareos y cuatro raleos. El primer ciclo sería de 8 años desde la condición inicial del bosque en que se aplica el primer clareo y hasta el segundo. Los ciclos posteriores van siendo cada vez más largos, siendo de 16 años el último (cuadro 5).

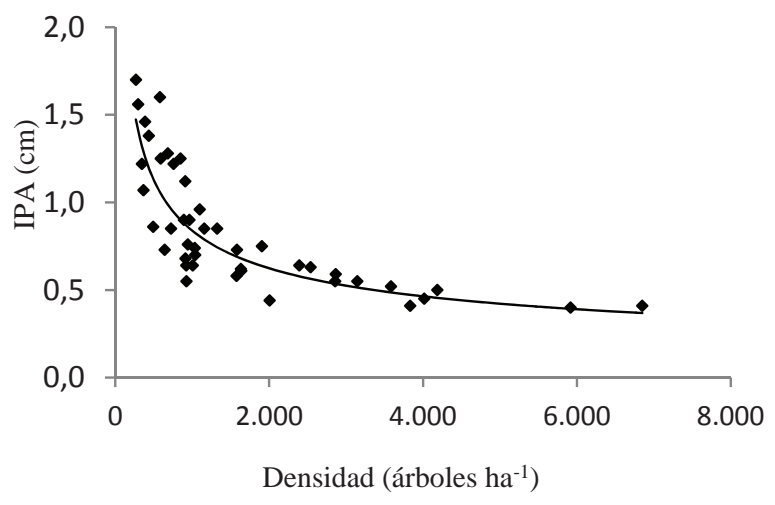

Figura 3. Distribución de los incrementos diametrales según densidad, usados para la función de incremento diametral de Nothofagus dombeyi.

Distribution of diametric growth by density, used for the increased diametric function for Nothofagus dombeyi.

La suma del volumen extraído en cada intervención y del volumen residual a la edad de 86 años (DAP de $50 \mathrm{~cm}$ ), fue de $1.217 \mathrm{~m}^{3} \mathrm{ha}^{-1}$, lo que representa un crecimiento volumétrico anual medio para el bosque $\left(\mathrm{CAM}_{\mathrm{vol}}\right)$ de 14,1 $\mathrm{m}^{3}$ año ha-1 ${ }^{-1}$ Estas cifras representarían la máxima posibilidad en volumen para una silvicultura ajustada a los mejores crecimientos del bosque. La configuración de intervenciones propuesta permitiría establecer que los tratamientos intermedios para este tipo de bosques, tendrían una duración en torno a 50 años (considerando un bosque inicial con 
Cuadro 3. Incrementos anuales y proyección de edad a la que se alcanza el diámetro clase, según densidad del bosque. Annual growth and projected age at which the diameter class is reached, according to forest density.

\begin{tabular}{ccccccc}
\hline \multicolumn{3}{c}{ Estimación de incrementos $\left(\mathrm{cm} \mathrm{año}^{-1}\right)$} & \multicolumn{3}{c}{ Edad (años) } \\
\hline Clase $(\mathrm{cm})$ & $\mathrm{IPA}_{100 \%}$ & $\mathrm{IPA}_{60 \%}$ & $\mathrm{IPA}_{35 \%}$ & $\mathrm{E}_{\text {IDR 100\% }}$ & $\mathrm{E}_{\text {IDR } 60 \%}$ & $\mathrm{E}_{\text {IDR } 35 \%}$ \\
\hline 5 & 0,19 & 0,24 & 0,30 & 26,1 & 21,0 & 16,6 \\
10 & 0,31 & 0,38 & 0,48 & 52,1 & 41,9 & 33,3 \\
15 & 0,41 & 0,51 & 0,64 & 68,3 & 54,9 & 43,6 \\
20 & 0,5 & 0,62 & 0,78 & 80,6 & 64,8 & 51,5 \\
25 & 0,58 & 0,72 & 0,91 & 90,7 & 72,9 & 57,9 \\
30 & 0,66 & 0,82 & 1,03 & 99,3 & 79,8 & 63,4 \\
35 & 0,73 & 0,91 & 1,14 & 107,0 & 86,0 & 68,3 \\
40 & 0,80 & 0,99 & 1,25 & 113,8 & 91,5 & 72,7 \\
45 & 0,87 & 1,08 & 1,36 & 120,1 & 96,5 & 76,7 \\
50 & 0,93 & 1,16 & 1,46 & 125,9 & 101,2 & 80,4 \\
\hline
\end{tabular}

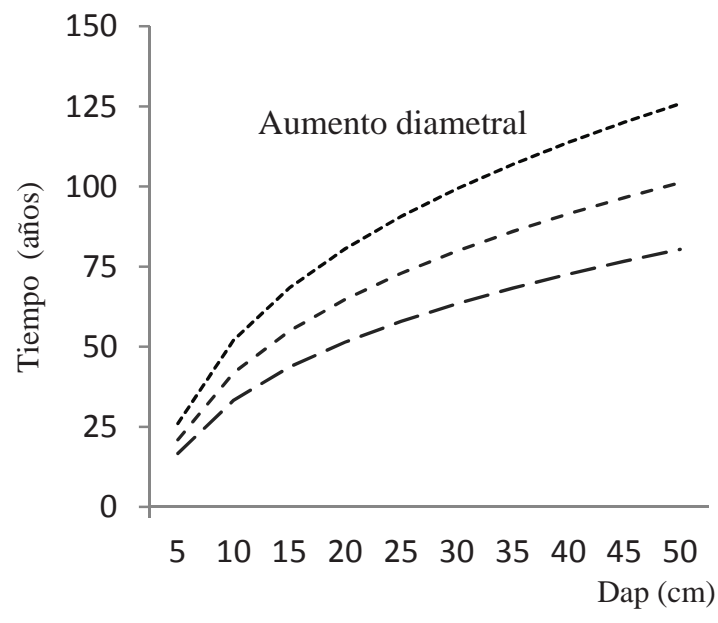

Figura 4. Aumentos del diámetro a través del tiempo para las tres condiciones de densidad analizadas.

Diametric growth over time for the three densities conditions analyzed.

edad en torno a 20 años). La evolución de las intervenciones y la variación del volumen residual a lo largo del tiempo (figura 5) significan reducciones de densidad en un rango del 49 al $46 \%$ del total en los clareos y el 39 y $45 \%$ en los raleos (figura 6). La consecuencia en el crecimiento diametral es un aumento homogéneo desde un valor inicial de $0,25 \mathrm{~cm}$ año ${ }^{-1}$ hasta un valor de $1,36 \mathrm{~cm}$ año ${ }^{-1}$ aproximadamente a la edad de 86 años (figura 7).

\section{DISCUSIÓN}

Densidad puntual e índices de densidad del rodal (IDR). Los primeros antecedentes relativos a estimaciones de la densidad en bosques de coihue corresponden a ensayos realizados en la reserva forestal Malancahuello instalados en 1977 (Lara et al. 2000), y que describen densidades comprendidas entre los 1.600 árboles ha ${ }^{-1}$ y los 5.460 árboles $\mathrm{ha}^{-1}$ para diámetros medios cuadráticos de 19 y 8,5, respectivamente. Ambas cifras son equivalentes a los valores de densidad en el nivel $\mathrm{DN}_{60 \%}$ propuestos en este estudio. Delgado (1986), en su caracterización de renovales de $N$. dombeyi en la laguna Pedro Aguirre Cerda XI, reportó densidades de 1.927 árboles ha- ${ }^{-1}$ y DAP de 17,7 cm, similares a las encontradas en este estudio. Así también, Donoso et al. (1999), en renovales de $N$. dombeyi de la Cordillera de la Costa y la de Los Andes, encontraron densidades de 5.425 árboles ha $^{-1}$ para el renoval andino y de 3.146 árboles ha-1 para el renoval costero, con diámetros medios cuadráticos de 10,5 y 13,5, respectivamente, y que se corresponderían a densidades estimadas a partir del área potencial aprovechable (APA) a densidades comprendidas en el rango $\mathrm{DN}_{60 \%} \mathrm{y} \mathrm{DN}_{100 \%}$. Los resultados anteriores indican que el método del área potencialmente aprovechable describe densidades similares a las encontradas por otros autores y estima satisfactoriamente la densidad en $N$. dombeyi, con la ventaja de asegurar que las mediciones provienen siempre de porciones puras de bosque, mejorando los inconvenientes de asignar atributos de un individuo la densidad promedio de todo un rodal, o bien aceptar parcelas con presencia de otras especies.

Respecto del uso de índices de densidad, a la fecha de esta publicación existen escasos trabajos para Nothofagus spp. en Chile. Gezan et al. (2007), estimó un índice de densidad del rodal máximo ( IDR $_{\max }$ ) de 2.239 para $N$. dombeyi, valor muy similar al encontrado en este estudio para la misma especie (2.387). Para otras especies del género a las que se les ha determinado el valor de $I_{\text {IDR }}$, se han establecido cifras sustantivamente diferentes. Chauchard et al. (2001), en bosques mixtos de Nothofagus spp. en 
Cuadro 4. Estimación de las principales variables del rodal antes y después de cada intervención para el esquema silvícola. Estimation of the main variables of the stand before and after each intervention to silvicultural scheme.

\begin{tabular}{|c|c|c|c|c|c|c|c|c|}
\hline Años & $\begin{array}{l}\text { Edad } \\
\text { (años) }\end{array}$ & $\begin{array}{l}\text { DAP Clase } \\
(\mathrm{cm})\end{array}$ & $\begin{array}{c}\mathrm{DN}_{\mathrm{IDR}} \\
\left(\text { (árboles } \text { ha }^{-1} \text { ) }\right.\end{array}$ & $\begin{array}{l}\text { IDR } \\
(\%)\end{array}$ & $\begin{array}{c}\text { Incrementos dimétricos (cm) } \\
\text { obtenidos de la fórmula [6] }\end{array}$ & $\begin{array}{l}\text { Volumen acumulado } \\
\qquad\left(\mathrm{m}^{3} \mathrm{ha}^{-1}\right)\end{array}$ & $\begin{array}{l}\text { Actividad } \\
\text { silvícola }\end{array}$ & $\begin{array}{l}\text { Extracción } \\
\quad\left(\mathrm{m}^{3}\right)\end{array}$ \\
\hline 0 & 22 & 5 & 20.542 & 65 & 0,24 & 135,36 & & \\
\hline 1 & 23 & 5,2 & 10.400 & 35 & 0,30 & 77,04 & clareo 1 & 58 \\
\hline 2 & 24 & 5,5 & 10.296 & 38 & 0,31 & 87,60 & & \\
\hline 3 & 25 & 5,9 & 10.193 & 41 & 0,31 & 99,19 & & \\
\hline 4 & 26 & 6,2 & 10.091 & 45 & 0,31 & 111,54 & & \\
\hline 5 & 27 & 6,5 & 9.990 & 48 & 0,31 & 124,63 & & \\
\hline 6 & 28 & 6,8 & 9.890 & 51 & 0,32 & 138,46 & & \\
\hline 7 & 29 & 7,1 & 9.791 & 54 & 0,32 & 153,04 & & \\
\hline 8 & 30 & 7,4 & 9.693 & 58 & 0,32 & 168,35 & & \\
\hline 9 & 31 & 7,7 & 5.500 & 35 & 0,40 & 105,68 & clareo 2 & 63 \\
\hline 10 & 32 & 8,1 & 5.445 & 38 & 0,41 & 118,26 & & \\
\hline 11 & 33 & 8,5 & 5.391 & 40 & 0,41 & 131,57 & & \\
\hline 12 & 34 & 9,0 & 5.337 & 43 & 0,41 & 145,59 & & \\
\hline 13 & 35 & 9,4 & 5.283 & 46 & 0,41 & 160,33 & & \\
\hline 14 & 36 & 9,8 & 5.230 & 49 & 0,41 & 175,78 & & \\
\hline 15 & 37 & 10,2 & 5.178 & 51 & 0,42 & 191,94 & & \\
\hline 16 & 38 & 10,6 & 5.126 & 54 & 0,42 & 208,80 & & \\
\hline 17 & 39 & 11,0 & 5.075 & 57 & 0,42 & 226,36 & & \\
\hline 18 & 40 & 11,4 & 5.024 & 60 & 0,42 & 244,61 & & \\
\hline 19 & 41 & 11,9 & 2.750 & 35 & 0,54 & 145,71 & raleo 1 & 99 \\
\hline 20 & 42 & 12,4 & 2.723 & 37 & 0,55 & 160,21 & & \\
\hline 21 & 43 & 13,0 & 2.695 & 39 & 0,55 & 175,39 & & \\
\hline 22 & 44 & 13,5 & 2.668 & 42 & 0,55 & 191,25 & & \\
\hline 23 & 45 & 14,1 & 2.642 & 44 & 0,55 & 207,79 & & \\
\hline 24 & 46 & 14,6 & 2.615 & 46 & 0,56 & 224,99 & & \\
\hline 25 & 47 & 15,2 & 2.589 & 49 & 0,56 & 242,86 & & \\
\hline 26 & 48 & 15,7 & 2.563 & 51 & 0,56 & 261,38 & & \\
\hline 27 & 49 & 16,3 & 2.538 & 53 & 0,56 & 280,56 & & \\
\hline 28 & 50 & 16,9 & 2.512 & 56 & 0,57 & 300,38 & & \\
\hline 29 & 51 & 17,4 & 2.487 & 58 & 0,57 & 320,85 & & \\
\hline 30 & 52 & 18,0 & 1.400 & 35 & 0,73 & 194,43 & raleo 2 & 126 \\
\hline 31 & 53 & 18,7 & 1.386 & 36 & 0,73 & 210,78 & & \\
\hline 32 & 54 & 19,4 & 1.372 & 38 & 0,73 & 227,77 & & \\
\hline 33 & 55 & 20,2 & 1.358 & 40 & 0,74 & 245,38 & & \\
\hline 34 & 56 & 20,9 & 1.345 & 42 & 0,74 & 263,63 & & \\
\hline 35 & 57 & 21,6 & 1.331 & 44 & 0,74 & 282,49 & & \\
\hline 36 & 58 & 22,4 & 1.318 & 46 & 0,75 & 301,97 & & \\
\hline 37 & 59 & 23,1 & 1.305 & 48 & 0,75 & 322,05 & & \\
\hline 38 & 60 & 23,9 & 1.292 & 50 & 0,75 & 342,74 & & \\
\hline 39 & 61 & 24,6 & 1.279 & 52 & 0,76 & 364,02 & & \\
\hline 40 & 62 & 25,4 & 1.266 & 54 & 0,76 & 385,89 & & \\
\hline 41 & 63 & 26,1 & 1.253 & 56 & 0,76 & 408,34 & & \\
\hline 42 & 64 & 26,9 & 1.241 & 59 & 0,76 & 431,37 & & \\
\hline 43 & 65 & 27,7 & 700 & 35 & 0,98 & 259,23 & raleo 3 & 172 \\
\hline
\end{tabular}




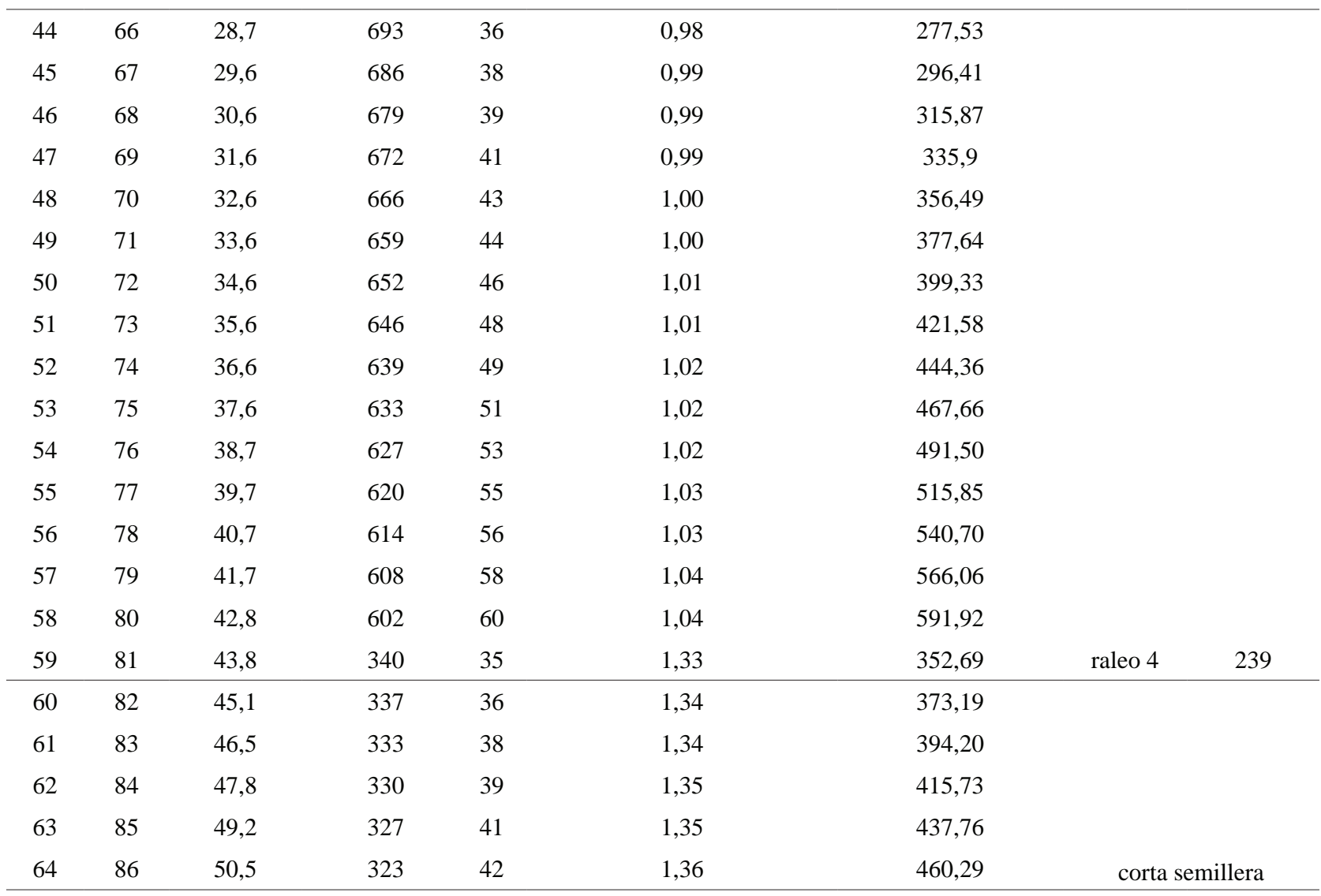

Cuadro 5. Propuesta de esquema silvícola para Nothofagus dombeyi en la cuenca del río Mirta. Proposal silvicultural scheme by Nothofagus dombeyi in the Mirta river basin.

\begin{tabular}{|c|c|c|c|c|c|c|c|}
\hline \multirow[t]{2}{*}{ Años } & \multirow[t]{2}{*}{ Edad (años) } & \multirow{2}{*}{$\begin{array}{c}\text { Clase de } \\
\text { DAP }(\mathrm{cm})\end{array}$} & \multirow{2}{*}{$\begin{array}{l}\text { Volumen ha-1 } \\
\qquad\left(\mathrm{m}^{3}\right)\end{array}$} & \multirow[t]{2}{*}{ Intervención } & \multicolumn{2}{|c|}{ Densidad (árboles ha-1) } & \multirow[t]{2}{*}{ Extracción } \\
\hline & & & & & Inicial & Final & \\
\hline 1 & 23 & 5 & 135 & Clareo 1 & 20.542 & 10.400 & 58 \\
\hline 9 & 31 & 8 & 168 & Clareo 2 & 9.693 & 5.500 & 63 \\
\hline 19 & 41 & 12 & 244 & Raleo 1 & 5.024 & 2.750 & 99 \\
\hline 30 & 52 & 18 & 320 & Raleo 2 & 2.487 & 1.400 & 126 \\
\hline 43 & 65 & 28 & 431 & Raleo 3 & 1.241 & 700 & 172 \\
\hline 59 & 81 & 44 & 592 & Raleo 4 & 602 & 340 & 239 \\
\hline
\end{tabular}

la Patagonia mencionan valores de 1.422, Martínez et al. (2002) para bosques de Nothofagus pumilio (Poepp. et Endl.) Krasser, en Santa Cruz y Tierra del Fuego propone 1.391, y Müller et al. (2013) para robles de la zona del Biobío estimó el $\mathrm{IDR}_{\max }$ en 980.

Nótese que al evaluar un DAP idéntico al normal (frecuentemente $25 \mathrm{~cm}$ ), la expresión de Reineke hace equi-

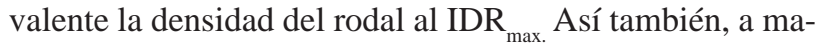
yores diámetros la función generará densidades menores y proporcionales al $\mathrm{IDR}_{\max }$. Matemáticamente también, menores diámetros implicarán un aumento exponencial de la densidad, resultando que diferencias pequeñas en el DAP significan miles de árboles por hectárea, determinados principalmente por el exponente de la función, más que por el máximo $\mathrm{IDR}_{\max }$.

Silvícolamente, el diámetro normal $(25 \mathrm{~cm})$ determina un inicio aproximado del estado de latizal y, en consecuencia, las actividades silvícolas programadas desde ese momento hasta la cosecha implican la selección de individuos como árboles para un producto final (Vita 1996, Lara et al. 1998, Navarro et al. 2011). El IDR max $_{\text {ax }}$ será el grupo de árboles que se dispondría para todas las actividades sil- 


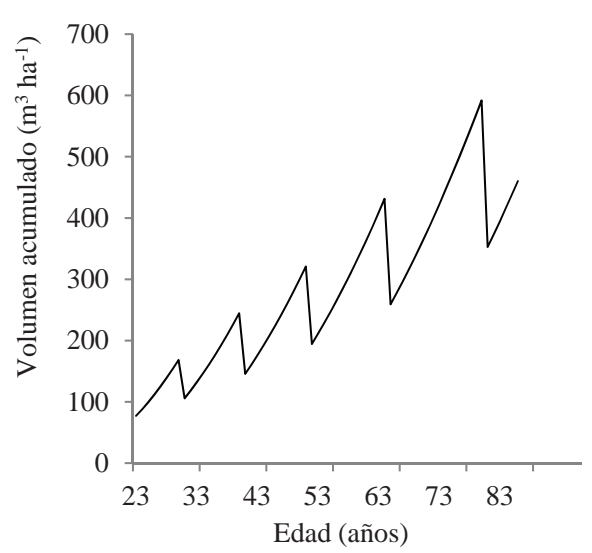

Figura 5. Variación en la acumulación de volumen con el tiempo, incluyendo los cortes indicados en el esquema de silvícola.

Variation in volume accumulation over time, including the cuttings indicated in de silvicultural scheme.

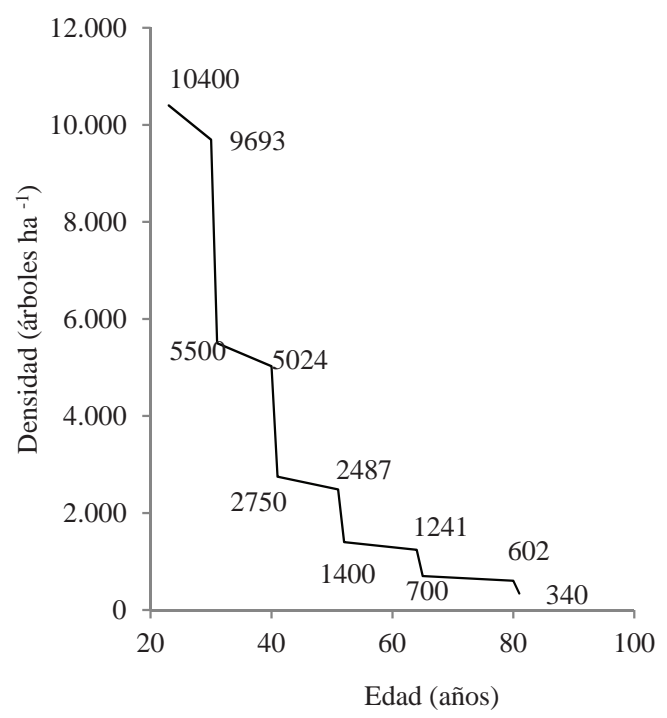

Figura 6. Diagrama de variación de la densidad causada por deferentes actividades del esquema silvicultural.

Density variation diagram caused by the different activities of silvicultural scheme.

vícolas futuras. Por ejemplo, para bosques de N. pumilio una densidad de 1.422 árboles ha-1 ${ }^{-1}$ si fuera el caso de las propuestas de Chauchard et al. (2001)

Así también, en estados de desarrollo menores al DAP normal, especialmente los estados de brinzal, la densidad puede estar fuertemente influenciada por factores diferentes a la mera competencia intraespecífica, como son la disponibilidad de semillas, los patógenos y los depredadores de la regeneración por mencionar algunos (Cruz 2013), lo que podría provocar diferencias significativas entre los datos reales de una situación y una formulación de relación diámetro/densidad como la de Reineke. A pesar de ello, las altas densidades propias de estos estados otorgan flexibili-

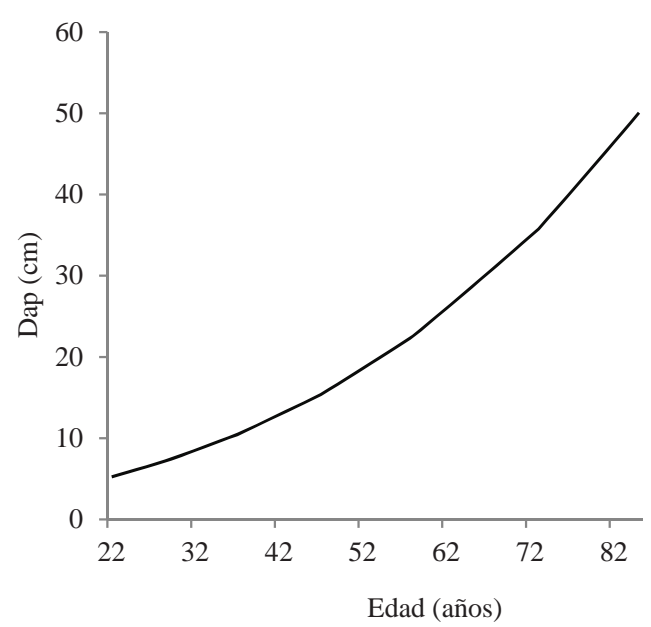

Figura 7. Crecimiento diametral según edad, para un bosque sometido a las intervenciones indicadas en el esquema silvícola.

Diametric growth by age, for a forest submitted to the cutting indicated in the silvicultural scheme.

dad a la silvicultura, sumado a que estos estados juveniles sólo requieren de actividades silvícolas sencillas de reducción de densidad, permitiendo afirmar que las deficientes estimaciones de los modelos serían menos relevantes que aquellas descritas para situaciones con valores superiores al diámetro normal. En consecuencia, en la aplicación de un índice de rodal de Reineke desde una perspectiva silvícola, parece más relevante la obtención de un $\mathrm{IDR}_{\max }$ consistente, que los efectos del exponente.

Propuesta de esquema de manejo silvícola. Los antecedentes silvícolas publicados para la especie son extremadamente escasos, y no se han encontrado publicaciones relativas a esquemas de manejo para coihue. En el caso de otras especies del género, y que habitan áreas compartidas con coihue, como son renovales de roble y raulí, Donoso (1988) propone intervenciones con magnitudes entre el 30 y el $45 \%$ de la densidad y entre el 25 y el $30 \%$ de área basal, llegando a la cosecha final con una densidad de 600 árboles ha ${ }^{-1}$ a edades entre 60 y 80 años, basándose en los crecimientos en diámetro y altura. Los baremos propuestos por los autores son relativamente similares a los que arroja el esquema propuesto en este estudio, especialmente las intensidades de extracción en densidad y las edades de rotación estimadas.

Un hecho que refleja el esfuerzo silvícola que implica el esquema de manejo, es que si se aplicaran las cortas en el momento y la intensidad recomendada, el diámetro normal fijado en $25 \mathrm{~cm}$ debiera alcanzarse con una densidad en torno a 1.250 árboles ha ${ }^{-1}$ a los 62 años, dicha densidad representaría el $54 \%$ de la máxima posible para ese estado, un incremento diametral de $0,76 \mathrm{~cm}^{2} \mathrm{ano}^{-1}$, y ya habría recibido cuatro intervenciones de reducción de densidad. En cambio sin silvicultura, a los $25 \mathrm{~cm}$ de diámetro eventualmente podría haber alcanzado los 2.387 árboles 
hectárea $^{-1}$ con crecimientos sustantivamente menores, en un plazo más largo y con reducciones de densidad debidas mayoritariamente a autorraleos.

El volumen total acumulado estimado para la especie en la zona de estudio $\left(1.217 \mathrm{~m}^{3} \mathrm{ha}^{-1}\right.$ en 86 años y un crecimiento anual medio de $14 \mathrm{~m}^{3}$ año ha-1), es elevado en comparación con bosques latifoliados manejados de Europa. Los datos publicados por Diéguez-Aranda (2009) para Galicia, indican valores de 7 y $10 \mathrm{~m}^{3}$ año ha-1, para Betula alba L. y Quercus robur L., respectivamente. Rodríguez y Vega (1998), para Fagus sylvatica L. indican crecimientos medios de volumen a final de la rotación de $11,8 \mathrm{~m}^{3}$ año ha1. Estas cifras confirma que los bosques nativos chilenos, al menos los de la especie estudiada, tienen un crecimiento sustantivamente mayor a los bosques templados europeos.

\section{CONCLUSIONES}

El método propuesto para el análisis de diámetro/densidad, consistente en usar la combinación APA como estimador de densidad, y el índice de Reineke para definir las máximas densidades posibles, dio resultados satisfactorios, validados por las diversas caracterizaciones cuantitativas que han hecho otros autores. Particularmente usar el APA como estimador de densidad ofrece las ventajas de ser simple y de bajo costo, garantizar la captura de información en porciones puras del bosque y mejorar la relación del atributo medido del árbol con su condición de densidad.

El esquema propuesto fue posible gracias a la aplicación de tres algoritmos: el índice de Reineke, aplicado con su exponente original, los límites de densidad de Long y la función de incremento diametral dependiente de densidad. Si bien diversos autores han recomendado tipos e intensidades de intervención para la especie, la ventaja de la construcción de un esquema silvícola como herramienta operativa es que contiene la dinámica para toda una rotación y puede utilizarse para cualquier condición de bosque de la especie, respecto de su estado de desarrollo y densidad.

Así, sería recomendable que los resultados de este trabajo, especialmente la metodología empleada fuera ensayada en otras formaciones boscosas dominadas por Nothofagus spp., que actualmente en Chile presentan una importante superficie de bosques, para los cuales se podría reducir la complejidad y los costos del manejo.

\section{REFERENCIAS}

Adame P, J Hynynen, C Isabel, M Del Río. 2008. Individual tree diameter growth model for rebollo oak (Quercus pyrenaica). Forest Ecology Management 255: 1011-1022.

Brown G. 1965. Point density in stems per acre. New Zeland Forest Research 38: 2-15.

Castedo F, U Diéguez-Aranda, A Rojo, JG Álvarez. 2009. Herramientas selvícolas para la gestión forestal sostenible en Galicia. Lugo, España. Xunta de Galicia. 250 p.

Chauchard L, R Sbrancia, M González, L Maresca, A Rabino, M Mazzuchelli. 2001. Aplicación de leyes fundamentales de la densidad a bosques de Nothofagus: II. Línea de inicio de competencia y diagramas de manejo de la densidad. Bosque 22 (1): 3- 10.

Cottam G, JT Curtis. 1956. The use of distance measure in phytosociological sampling. Ecology 30: 271-287.

Cruces P, J Cerda, M Ahumada. 1999. Guías de Condición para los Pastizales de la Ecorregión Templada Húmeda de Aysén. Proyecto FNDR-SAG XI Región de Aysén. 137 p.

Cruz P. 2011. Aplicaciones de ArcInfo para investigación en silvicultura: análisis fustal y Área Potencial Aprovechable. Consultado 4 dic. 2011. Disponible en http://www.usuarios. cl/presentaciones/Aplicaciones\%20Oterra1.pdf

Cruz P. 2013. Aplicación de relaciones de densidad-diámetro y de área potencial aprovechable en bosques de Nothofagus dombeyi (Mirb.) Oerst, como herramienta de planificación silvícola. Tesis doctoral. Albacete, España. Universidad de Castilla La Mancha. 105 p.

Delgado C. 1986. Caracterización del renoval de coihue (Nothofagus dombeyi (Mirb.) Oerst.) en el sector Laguna Pedro Aguirre Cerda-Monte Picaflor, XI Región de Aysén. Tesis Ingeniero Forestal. Concepción Chile. Facultad de ciencias Forestales. Universidad de Concepción. 56 p.

Diéguez-Aranda U, A Rojo, F Castedo, JG Álvarez. 2009. Herramientas selvícolas para la gestión forestal sostenible en Galicia. Lugo, España. Xunta de Galicia. 250 p.

Donoso P, C Cabezas, A Lavanderos, C Donoso. 1999. Desarrollo de renovales de coihue común (Nothofagus dombeyi (Mirb.) Oerst.) en la Cordillera de la Costa y de los andes de la provincia de Valdivia en sus primeros 25 años. Bosque 20(2): 9-23.

Donoso P. 1988. Caracterización y proposiciones silviculturales para renovales de roble (Nothofagus obliqua) y raulí (Nothofagus alpina) en el área de protección "Radal 7 tazas”, VII Región. Bosque 9(2): 109-114.

Drake F, P Emanuelli, E Acuña. 2003. Compendio de funciones dendrométricas del Bosque Nativo. Santiago, Chile. CONAF y GTZ. 197 p.

Esse C, C Navarro, JC Pinares. 2007. Curvas de índice de sitio para Nothofagus dombeyi en la zona preandina, provincia de cautín, IX región, Chile. Bosque 28(2): 142-151.

Gezan SA, A Ortega, E Andenmatten. 2007. Diagramas de manejo de la densidad para renovales de roble, raulí y coigüe en Chile. Bosque 28(2): 97-105.

Gingrich SF. 1967. Measuring and evaluating stocking and stand density in Upland Hardwood forests in the Central States. Forest Science 13(1): 38-53.

Lara A, C Donoso, P Donoso, P Nuñez, A Cavieres. 1998. Normas de manejo para raleo de renovales del tipo forestal roble-raulí-coigüe. Silvicultura de los Bosques Nativos de Chile. Santiago, Chile. Editorial Universitaria. p. 12-144.

Lara A, C Echeverría, C Donoso. 2000. Guía de ensayos silviculturales permanentes en los bosques nativos de Chile. Santiago, Chile. LOM. 280 p.

Long J. 1985. A practical approach to density management. Forestry Chronicle 61: 23-27.

Martínez G, MV Lencinas, JM Celleni, B Diaz, R Vukasovic. 2002. Herramientas disponibles para la construcción de un modelo de producción para la lenga (Nothofagus pumilio) bajo manejo en un gradiente de calidades de sitio. Bosque 23(2): 69-80.

Müller B, R Rodríguez, P Gajardo. 2013. Desarrollo de una guía 
de manejo de la densidad en bosques de segundo crecimiento de roble en la región del Bío Bío. Bosque 34(2): 201-209.

Navarro C, MA Herrerra, F Drake, P Donoso. 2011. Diagrama de manejo de densidad y su aplicación a raleo en bosques de segundo crecimiento de Drimys winteri en el sur de Chile. Bosque 32(2): 175-186.

Prodan M, R Peters, F Cox, P Real. 1997. Mensura Forestal. San José, Costa Rica. Iicc-Bmz/ Gtz. 586 p.

Reineke LH. 1933. Perfecting a stand density index for even aged forest. Journal Agricultural Research 46: 627-638.

Reque J. 2002. Prescripciones selvícolas para roble albar (Quercus petraea) en la Cordillera Cantábrica a partir de un análi- sis sincrónico. Investigacion Agraria 11(2): 284-297.

Rodríguez R, P Vega. 1998. Apuntes de silvicultura de zonas atlánticas. Lugo, España. Unicopia.758 p.

Vacchiano G, R Motta, J Long, J Shaw. 2008. A density management diagram for Scots pine (Pinus Sylvestris L.): A tools for assesing the forest's protective effect. Forestry Ecology and Management 255(7): 2542-2554.

Vita A. 1996. Los tratamientos silviculturales. Santiago, Chile. Universidad de Chile. 147 p.

Woodall C, P Miles, J Vissage. 2005. Determining maximun stand density index in mixed species stand for strategicscale stocking assessment. Forest Ecology and Management 216: 367-377.

Recibido: 02.01.14

Aceptado: 13.06.14 
